Bull. Korean Math. Soc. 52 (2015), No. 3, pp. 761-769

http://dx.doi.org/10.4134/BKMS.2015.52.3.761

\title{
SOME CHARACTERIZATIONS OF CHARACTER AMENABLE BANACH ALGEBRAS
}

\author{
Madjid Eshaghi Gordji, Ali Jabbari, And Gwang Hui Kim
}

\begin{abstract}
In this study, the character amenability of Banach algebras is considered and some characterization theorems are established. Indeed, we prove that the character amenability of Lipschitz algebras is equivalent to that of Banach algebras.
\end{abstract}

\section{Introduction}

The notion of amenability for Banach algebras was introduced and studied by Johnson [12]. He proved that for every locally compact group $G$, the group algebra $L^{1}(G)$ is amenable if and only if $G$ is amenable. Several generalizations and modifications of amenability have been considered and defined by numerous authors; see, for example, Ghahramani and Loy [6] and Grønbæk [9]. Let $\mathfrak{A}$ be a Banach algebra and $\sigma(\mathfrak{A})$ be the carrier space of $\mathfrak{A}$, and let $\varphi \in \sigma(\mathfrak{A})$ be a homomorphism from $\mathfrak{A}$ onto $\mathbb{C}$. The notion of character amenability of Banach algebras was defined by Monfared in [18]. Meanwhile, the concept of $\varphi$-amenability of Banach algebras was introduced by Kaniuth and et al. in [13]. These concepts were related to those cited in the work of Professor Lau in [16].

Assuming that $\varphi \in \sigma(\mathfrak{A}) \cup\{0\}$ and $X$ is an arbitrary Banach space, then $X$ can be viewed as Banach left or right $\mathfrak{A}$-module by the following actions:

$$
a \cdot x=\varphi(a) x \quad \text { and } \quad x \cdot a=\varphi(a) x \quad(a \in \mathfrak{A}, x \in X) .
$$

The Banach algebra $\mathfrak{A}$ is said to be left character amenable (LCA) if for all $\varphi \in \sigma(\mathfrak{A}) \cup\{0\}$ and all Banach $\mathfrak{A}$-bimodules $X$ for which the right module action is given by $a \cdot x=\varphi(a) x(a \in \mathfrak{A}, x \in X)$, every continuous derivation $D: \mathfrak{A} \longrightarrow X^{*}$ is inner. Meanwhile, right character amenability (RCA) is defined in a similar manner by considering Banach $\mathfrak{A}$-bimodules $X$ for which the left module action is given by $x \cdot a=\varphi(a) x$. Finally, $\mathfrak{A}$ is called character amenable $(\mathrm{CA})$ if it is both left and right character amenable.

Also, according to [13], the Banach algebra $\mathfrak{A}$ is $\varphi$-amenable $(\varphi \in \sigma(\mathfrak{A}))$ if there exists a bounded linear functional $m$ on $\mathfrak{A}^{*}$ satisfying $m(\varphi)=1$ and

Received November 24, 2013; Revised May 1, 2014.

2010 Mathematics Subject Classification. Primary 46H20, 46H25; Secondary 43 A20.

Key words and phrases. amenability, Banach algebra, character amenability. 
$m(f \cdot a)=\varphi(a) m(f)$ for all $a \in \mathfrak{A}$ and $f \in \mathfrak{A}^{*}$. Therefore, the Banach algebra $\mathfrak{A}$ is $\mathrm{CA}$ if and only if $\mathfrak{A}$ is $\varphi$-amenable, for every $\varphi \in \sigma(\mathfrak{A}) \cup\{0\}$.

Recently character amenability of various classes of Banach algebras considered, for instance Fourier-Stieltjes algebras and Fourier algebras studied in [11], abstract Segal algebras and Segal algebras studied in [2], and semigroup algebras in [5]. Similar to the amenability of Banach algebras, versions of CA such as character contractibility and essential character amenability, have been defined and studied (see $[2,11,19]$ ). Moreover, new results related to CA of Banach algebras have been reported in [17].

Let $\mathfrak{A}$ be a Banach algebra, and $\varphi \in \sigma(\mathcal{A})$. The left (right) $\varphi$-approximate diagonal and $\varphi$-virtual diagonal for $\mathfrak{A}$ are defined by Z. Hu et al. in [11]. A left (right) $\varphi$-approximate diagonal for $\mathfrak{A}$ is a net $\left(m_{\alpha}\right)$ in $\mathfrak{A} \widehat{\otimes} \mathfrak{A}$ such that

(i) $\left\|m_{\alpha} \cdot a-\varphi(a) m_{\alpha}\right\| \rightarrow 0\left(\left\|a \cdot m_{\alpha}-\varphi(a) m_{\alpha}\right\| \rightarrow 0\right)$ for every $a \in \mathfrak{A}$;

(ii) $\left\langle\varphi \otimes \varphi, m_{\alpha}\right\rangle=\varphi\left(\pi\left(m_{\alpha}\right)\right) \rightarrow 1$.

An element $M$ of $(\mathfrak{A} \widehat{\otimes} \mathfrak{A})^{* *}$ is a left (right) $\varphi$-virtual diagonal for $\mathfrak{A}$ if

(i) $M \cdot a=\varphi(a) M(a \cdot M=\varphi(a) M)$ for every $a \in \mathfrak{A}$;

(ii) $\langle M, \varphi \otimes \varphi\rangle=\pi^{* *}(M) \varphi=1$.

According to Theorem 2.3 of [11], the existences of bounded $\varphi$-approximate diagonal and bounded $\varphi$-virtual diagonal for Banach algebra $\mathfrak{A}$ are equivalent to $\varphi$-amenability of $\mathfrak{A}$, and $\varphi$-amenability of $\mathfrak{A}$ implies that both of them.

In this paper, we present some characterization results for character amenable ( $\varphi$-amenable) Banach algebras similar to the characterization of amenable Banach algebras [15]. Moreover, we study the character amenability of Lipschitz algebras.

\section{Character amenability}

A foundation semigroup $S$ is said to have the Hahn-Banach theorem property if for every left action of $S$ on a Banach space $X$, if $\rho$ is a continuous invariant seminorm on $X$, and $\phi$ is a $\chi$-invariant linear functional on an invariant subspace $Y$ of $X$ such that $|\phi| \leq \rho$, then there exists a $\chi$-invariant extension $\psi$ of $\phi$ to $X$ such that $|\psi| \leq \rho$. Ghaffari in [5] studied character amenability of semigroup algebras. He proved that if the unital foundation locally compact semigroup $S$ has the Hahn-Banach theorem property, then it is LCA and converse is true when $\|m\|=1$, where $m$ is the linear functional defined on $M_{a}(S)^{*}$, [5, Theorem 6] ( $m$ is the linear functional defined in Section 1). By $B(X)$ we mean the space of all linear bounded operators from Banach space $X$ into itself. Now, we prove the main theorem of paper as follows.

Theorem 2.1. Let $\mathfrak{A}$ be a Banach algebra with carrier space $\sigma(\mathfrak{A})$ and $X$ be a Banach $\mathfrak{A}$-bimodule with the mentioned action. Let $\varphi \in \sigma(\mathfrak{A})$. Then, the following statements are equivalent:

(i) $\mathfrak{A}$ is $\varphi$-amenable; 
(ii) For any $\mathfrak{A}$-Banach submodule $Y$ of $X$, each linear functional in $\bigcap_{a \in \mathfrak{A}}\left\{y^{*} \in Y^{*} \mid a \cdot y^{*}=\varphi(a) y^{*}\right\}$ has an extension to a linear functional in $\bigcap_{a \in \mathfrak{A}}\left\{x^{*} \in X^{*} \mid a \cdot x^{*}=\varphi(a) x^{*}\right\}$;

(iii) There is a bounded projection from $X^{*}$ onto $\bigcap_{a \in \mathfrak{A}}\left\{x^{*} \in X^{*} \mid a \cdot x^{*}=\right.$ $\left.\varphi(a) x^{*}\right\}$ which commutes with any bounded linear operator from $X^{*}$ into $X^{*}$ commuting with the action of $\mathfrak{A}$ on $X$.

Proof. (i) $\Rightarrow($ ii): Since $X$ is a Banach $\mathfrak{A}$-bimodule, then the quotient Banach space $X / Y$ is a Banach $\mathfrak{A}$-bimodule by the following actions:

$$
a \cdot(x+Y)=\varphi(a)(x+Y) \text { and }(x+Y) \cdot a=x \cdot a+Y
$$

for every $a \in \mathfrak{A}$ and $x \in X$. Let $\alpha \in \bigcap_{a \in \mathfrak{A}}\left\{y^{*} \in Y^{*} \mid a \cdot y^{*}=\varphi(a) y^{*}\right\}$. Suppose that $\bar{\alpha} \in X^{*}$ is a extension of $\alpha$. It is known that $\mathbf{q}: Y^{\perp}=\left\{x^{*} \in\right.$ $X^{*} \mid\left\langle y, x^{*}\right\rangle=0$ for every $\left.y \in Y\right\} \longrightarrow(X / Y)^{*}$ is an $\mathfrak{A}$-module isometry and surjection mapping. Then, for every $a \in \mathfrak{A}$ and $y \in Y$, we have

$$
\begin{aligned}
\langle y, a \cdot \bar{\alpha}\rangle-\langle y, \bar{\alpha} \cdot a\rangle & =\langle y \cdot a, \bar{\alpha}\rangle-\langle a \cdot y, \bar{\alpha}\rangle \\
& =\langle y, a \cdot \bar{\alpha}-\varphi(a) \bar{\alpha}\rangle=0 .
\end{aligned}
$$

Therefore, $a \cdot \bar{\alpha}-\varphi(a) \bar{\alpha} \in Y^{\perp}$. Define $D: \mathfrak{A} \longrightarrow(X / Y)^{*}$ by $D(a)=\mathbf{q}(a \cdot \bar{\alpha}-$ $\varphi(a) \bar{\alpha}) . \quad D$ is clearly a bounded derivation. Since $\mathfrak{A}$ is $\varphi$-amenable, so there is a $t \in(X / Y)^{*}$ such that $D(a)=a \cdot t-t \cdot a$. Then there exists $\beta \in Y^{\perp}(\mathbf{q}$ is surjective) such that $D(a)=a \cdot \mathbf{q}(\beta)-\varphi(a) \mathbf{q}(\beta)$ for all $a \in \mathfrak{A}$. Now, set $\gamma=\bar{\alpha}-\beta$. Then, for every $a \in \mathfrak{A}$ and $y \in Y$, we have

$$
\langle y, a \cdot(\bar{\alpha}-\beta)-\varphi(a)(\bar{\alpha}-\beta)\rangle=0 .
$$
(ii)

This means that $\gamma \in \bigcap_{a \in \mathfrak{A}}\left\{x^{*} \in X^{*} \mid a \cdot x^{*}=\varphi(a) x^{*}\right\}$. Therefore, (i) implies

(ii) $\Rightarrow$ (iii): Consider the projective tensor product of $X^{*}$ and $X, X^{*} \widehat{\otimes} X$. It is a Banach $\mathfrak{A}$-bimodule by the following actions:

$$
(f \otimes x) \cdot a=f \otimes x \cdot a \quad \text { and } \quad a \cdot(f \otimes x)=f \otimes \varphi(a) x=\varphi(a) f \otimes x
$$

for all $a \in \mathfrak{A}, x \in X$ and all $f \in X^{*}$. We define the sets $H$ and $K$ as follows

$$
\begin{gathered}
H:=\varlimsup \\
\left.\quad f \in X^{*}, x \in X\right\}
\end{gathered}
$$

and

$$
K:=\varlimsup\left\{f \otimes x \mid f \in \bigcap_{a \in \mathfrak{A}}\left\{x^{*} \in X^{*} \mid a \cdot x^{*}=\varphi(a) x^{*}\right\}\right\},
$$

where " $\star$ " is module product on $B(X)$, and by $\overline{\text { lin }}$ we mean closed linear span. Let $Y$ be the closed linear span of $H$ and $K$. Then, according to the definitions of $H$ and $K$, it is easy to see that $H$ and $K$ are Banach $\mathfrak{A}$-submodules of $X^{*} \widehat{\otimes} X$. 
Therefore, the quotient space $Y / H$ is a Banach $\mathfrak{A}$-submodule of $\left(X^{*} \widehat{\otimes} X\right) / H$. Let $\alpha \in\left(X^{*} \widehat{\otimes} X\right)^{*}$ satisfy $\langle f \otimes x, \alpha\rangle=f(x)$ for all $x \in X$ and all $f \in X^{*}$. Then

$$
\left\langle T^{*}(f) \otimes x-f \otimes T(x), \alpha\right\rangle=\left\langle x, T^{*}(f)\right\rangle-\langle T(x), f\rangle=0
$$

for all $x \in X$ and all $f \in X^{*}$. This means that $\alpha \in H^{\perp}$. Now, we get $\alpha^{\prime} \in\left(\left(X^{*} \widehat{\otimes} X\right) / H\right)^{*}$ such that $\alpha^{\prime}(y+H)=\alpha(y)$ for every $y \in X^{*} \widehat{\otimes} X$. First, we show that $a \cdot \alpha^{\prime}=\varphi(a) \alpha^{\prime}$ as a member of $(Y / H)^{*}$. It follows from $(2.1)$ and (2.2) that

$$
\begin{aligned}
& \left\langle f \otimes x+H, a \cdot \alpha^{\prime}\right\rangle-\left\langle f \otimes x+H, \alpha^{\prime} \cdot a\right\rangle \\
= & \left\langle(f \otimes x+H) \cdot a, \alpha^{\prime}\right\rangle-\left\langle a \cdot(f \otimes x+H), \alpha^{\prime}\right\rangle \\
= & \left\langle f \otimes x \cdot a+H, \alpha^{\prime}\right\rangle-\left\langle\varphi(a)(f \otimes x+H), \alpha^{\prime}\right\rangle \\
= & \langle f \otimes x \cdot a, \alpha\rangle-\langle\varphi(a)(f \otimes x), \alpha\rangle
\end{aligned}
$$

for all $a \in \mathfrak{A}, x \in X$ and all $f \in X^{*}$. By (2.3), $\alpha \in H^{\perp}$ so if we apply (2.4), we conclude that

$$
a \cdot \alpha^{\prime}=\varphi(a) \alpha^{\prime}
$$

for all $a \in \mathfrak{A}$ and all $\alpha^{\prime} \in(Y / H)^{*}$. By (ii), there is an extension $\widetilde{\alpha}$ of $\alpha^{\prime}$ such that $\widetilde{\alpha} \in(X / H)^{*}$ and $a \cdot \widetilde{\alpha}=\varphi(a) \widetilde{\alpha}$. Define $\langle x, P(f)\rangle=\langle f \otimes x+H, \widetilde{\alpha}\rangle$ for all $x \in X$ and all $f \in X^{*}$. Boundedness of $P$ is clear. Moreover, we have

$$
\begin{aligned}
\langle x, P \circ P(f)\rangle & =\langle x, P(P(f))\rangle \\
& =\langle P(f)(x), P\rangle \\
& =\langle(\langle f \otimes x+H, \widetilde{\alpha}\rangle), P\rangle \\
& =\langle f(x), P\rangle=\langle x, P(f)\rangle
\end{aligned}
$$

for all $x \in X$ and all $f \in X^{*}$. Hence, $P$ is a bounded projection from $X^{*}$ onto $\bigcap_{a \in \mathfrak{A}}\left\{x^{*} \in X^{*} \mid a \cdot x^{*}=\varphi(a) x^{*}\right\}$. Let $T \in \mathfrak{B}(X)$. Then $T^{*} \in \mathfrak{B}\left(X^{*}\right)$, and we have

$$
\left\langle x, P T^{*}(f)\right\rangle=\left\langle T^{*}(f) \otimes x, \widetilde{\alpha}\right\rangle=\left\langle x, T^{*}(f)\right\rangle
$$

$$
\begin{aligned}
& =\langle f \otimes T(x), \alpha\rangle=\langle f \otimes T(x)+H, \widetilde{\alpha}\rangle=\langle T(x), P(f)\rangle \\
& =\left\langle x, T^{*} P(f)\right\rangle
\end{aligned}
$$

for all $x \in X$ and all $f \in X^{*}$. Therefore, the obtained projection $P$ commutes with every $T^{*} \in \mathfrak{B}\left(X^{*}\right)$, which proves (iii).

Now, we prove that (iii) $\Longrightarrow$ (i). Without loss of generality, we suppose that $\mathfrak{A}$ is unital. Set $X=\mathfrak{A} \widehat{\otimes} \mathfrak{A}$, which $X$ is a Banach $\mathfrak{A}$-bimodule by the following actions:

$$
a \cdot(b \otimes c)=\varphi(a) b \otimes c \quad \text { and } \quad(b \otimes c) \cdot a=b \otimes c a
$$

for all $a, b, c \in \mathfrak{A}$. Let $\mathcal{F}=\left\{R_{a} \mid a \in \mathfrak{A}\right\} \cup\left\{L_{a} \mid a \in \mathfrak{A}\right\}$ be a family of bounded linear operators from $X$ into $X$, such that

$$
L_{a}(b \otimes c)=b \otimes \varphi(a) c \quad \text { and } \quad R_{a}(b \otimes c)=b a \otimes c
$$


for all $a, b, c \in \mathfrak{A}$. Then,

$$
b \cdot L_{a}(c \otimes d)=\varphi(b) L_{a}(c \otimes d) \quad \text { and } \quad b \cdot R_{a}(c \otimes d)=\varphi(b) R_{a}(c \otimes d)
$$

for all $a, b, c, d \in \mathfrak{A}$. This means that every member of $\mathcal{F}$ commutes with the actions of $\mathfrak{A}$ on $X$. So, by (iii), there exists a bounded surjective projection $P: X^{*} \longrightarrow X^{*}$ such that $P T^{*}=T^{*} P$ for all $T \in \mathcal{F}$. Define $q: X^{*} \longrightarrow X^{*}$ by $\langle a \otimes b, q(f)\rangle=\langle b \otimes a, f\rangle$ for all $a, b \in \mathfrak{A}$ and all $f \in X^{*}$. Set $M=q^{*}\left(P^{*}\left(e_{\mathfrak{A}} \otimes\right.\right.$ $\left.e_{\mathfrak{A}}\right)$ ). We claim that $M$ is a bounded left $\varphi$-virtual diagonal. According to the definition of $q$, we have

$$
\begin{aligned}
\left\langle c \otimes d, q\left(x^{*} \cdot a\right)\right\rangle & =\left\langle d \otimes c, x^{*} \cdot a\right\rangle=\left\langle\varphi(a) d \otimes c, x^{*}\right\rangle=\left\langle c \otimes \varphi(a) d, q\left(x^{*}\right)\right\rangle \\
& =\left\langle L_{a}(c \otimes d), q\left(x^{*}\right)\right\rangle=\left\langle c \otimes d, L_{a}^{*} q\left(x^{*}\right)\right\rangle
\end{aligned}
$$

for all $a, b, c, d \in \mathfrak{A}$ and all $x * \in X^{*}$. It follows that

$$
\begin{aligned}
\left\langle c \otimes d, q\left(a \cdot x^{*}\right)\right\rangle & =\left\langle d \otimes c, a \cdot x^{*}\right\rangle=\left\langle d \otimes c a, x^{*}\right\rangle=\left\langle c a \otimes d, q\left(x^{*}\right)\right\rangle \\
& =\left\langle R_{a}(c \otimes d), q\left(x^{*}\right)\right\rangle=\left\langle c \otimes d, R_{a}^{*} q\left(x^{*}\right)\right\rangle
\end{aligned}
$$

for all $a, b, c, d \in \mathfrak{A}$ and all $x * \in X^{*}$. Then, through the properties of $P$ and relation $(2.9)$, we have

$$
\begin{aligned}
\left\langle x^{*}, M \cdot a\right\rangle & =\left\langle a \cdot x^{*}, q^{*}\left(P^{*}\left(e_{\mathfrak{A}} \otimes e_{\mathfrak{A}}\right)\right)\right\rangle=\left\langle q\left(a \cdot x^{*}\right), P^{*}\left(e_{\mathfrak{A}} \otimes e_{\mathfrak{A}}\right)\right\rangle \\
& =\left\langle R_{a}^{*} q\left(x^{*}\right), P^{*}\left(e_{\mathfrak{A}} \otimes e_{\mathfrak{A}}\right)\right\rangle=\left\langle P R_{a}^{*} q\left(x^{*}\right), e_{\mathfrak{A}} \otimes e_{\mathfrak{A}}\right\rangle \\
& =\left\langle R_{a}^{*} P q\left(x^{*}\right), e_{\mathfrak{A}} \otimes e_{\mathfrak{A}}\right\rangle=\left\langle P q\left(x^{*}\right), R_{a}\left(e_{\mathfrak{A}} \otimes e_{\mathfrak{A}}\right)\right\rangle \\
& =\left\langle P q\left(x^{*}\right), a \otimes e_{\mathfrak{A}}\right\rangle=\varphi(a)\left\langle P q\left(x^{*}\right), e_{\mathfrak{A}} \otimes e_{\mathfrak{A}}\right\rangle \\
& =\varphi(a)\left\langle q\left(x^{*}\right), P^{*}\left(e_{\mathfrak{A}} \otimes e_{\mathfrak{A}}\right)\right\rangle=\varphi(a)\left\langle x^{*}, q^{*}\left(P^{*}\left(e_{\mathfrak{A}} \otimes e_{\mathfrak{A}}\right)\right)\right\rangle \\
& =\left\langle x^{*}, \varphi(a) M\right\rangle
\end{aligned}
$$

for all $x^{*} \in X^{*}$. Similarly, by (2.8), we have

$$
\begin{aligned}
\left\langle x^{*}, a \cdot M\right\rangle & =\left\langle x^{*} \cdot a, q^{*}\left(P^{*}\left(e_{\mathfrak{A}} \otimes e_{\mathfrak{A}}\right)\right)\right\rangle=\left\langle q\left(x^{*} \cdot a\right), P^{*}\left(e_{\mathfrak{A}} \otimes e_{\mathfrak{A}}\right)\right\rangle \\
& =\left\langle L_{a}^{*} q\left(x^{*}\right), P^{*}\left(e_{\mathfrak{A}} \otimes e_{\mathfrak{A}}\right)\right\rangle=\left\langle P L_{a}^{*} q\left(x^{*}\right), e_{\mathfrak{A}} \otimes e_{\mathfrak{A}}\right\rangle \\
& =\left\langle L_{a}^{*} P q\left(x^{*}\right), e_{\mathfrak{A}} \otimes e_{\mathfrak{A}}\right\rangle=\left\langle P q\left(x^{*}\right), L_{a}\left(e_{\mathfrak{A}} \otimes e_{\mathfrak{A}}\right)\right\rangle \\
& =\left\langle P q\left(x^{*}\right), e_{\mathfrak{A}} \otimes a\right\rangle=\varphi(a)\left\langle P q\left(x^{*}\right), e_{\mathfrak{A}} \otimes e_{\mathfrak{A}}\right\rangle \\
& =\varphi(a)\left\langle q\left(x^{*}\right), P^{*}\left(e_{\mathfrak{A}} \otimes e_{\mathfrak{A}}\right)\right\rangle=\varphi(a)\left\langle x^{*}, q^{*}\left(P^{*}\left(e_{\mathfrak{A}} \otimes e_{\mathfrak{A}}\right)\right)\right\rangle \\
& =\left\langle x^{*}, \varphi(a) M\right\rangle
\end{aligned}
$$

for all $x^{*} \in X^{*}$. Therefore, (2.10) and (2.11) imply that $a \cdot M=\varphi(a) M=M \cdot a$. Also, we have

$$
\begin{aligned}
\left\langle\varphi, \pi^{* *} M\right\rangle & =\left\langle\pi^{*} \varphi, q^{*}\left(P^{*}\left(e_{\mathfrak{A}} \otimes e_{\mathfrak{A}}\right)\right)\right\rangle=\left\langle q\left(\pi^{*} \varphi\right), P^{*}\left(e_{\mathfrak{A}} \otimes e_{\mathfrak{A}}\right)\right\rangle \\
& =\left\langle P\left(q\left(\pi^{*} \varphi\right)\right), e_{\mathfrak{A}} \otimes e_{\mathfrak{A}}\right\rangle=\left\langle q\left(\pi^{*} \varphi\right), e_{\mathfrak{A}} \otimes e_{\mathfrak{A}}\right\rangle \\
& =\left\langle\pi^{*} \varphi, e_{\mathfrak{A}} \otimes e_{\mathfrak{A}}\right\rangle=\left\langle\varphi, \pi^{* *}\left(e_{\mathfrak{A}} \otimes e_{\mathfrak{A}}\right)\right\rangle=\varphi\left(e_{\mathfrak{A}}\right)=1 .
\end{aligned}
$$


Note that we obtained the first equality on the third line by definition of $q$. Thus, $\pi^{* *}(M) \varphi=1$. Therefore, Theorem 2.3 of [11] implies that $\mathfrak{A}$ is $\varphi$-amenable.

By the following theorem, we give a strictly positive lower bounded between two characters of character amenable Banach algebras which is useful in characterization of character amenable Banach algebras.

Theorem 2.2. Let $\mathfrak{A}$ be a Banach algebra and let $\varphi, \psi \in \sigma(\mathfrak{A})$ such that $\varphi$ and $\psi$ are distinct. If $\mathfrak{A}$ is $\varphi$-and $\psi$-amenable, then there exists a positive number $M<\infty$ such that $\|\varphi-\psi\| \geq M \max (1,\|\varphi\|)^{-1}$.

Proof. Let $\mathfrak{A}$ be $\varphi$ and $\psi$-amenable. Then by Theorem 1.4 of [13], there exist bounded nets $\left(u_{\alpha}\right)$ and $\left(v_{\beta}\right)$ in $\mathfrak{A}$ such that $\left\|a \cdot u_{\alpha}-\varphi(a) u_{\alpha}\right\| \rightarrow 0$, $\left\|a \cdot v_{\beta}-\psi(a) v_{\beta}\right\| \rightarrow 0$ for all $a \in \mathfrak{A}$ and $\varphi\left(u_{\alpha}\right)=\psi\left(v_{\beta}\right)=1$ for all $\alpha, \beta$.

Consider the net $\left(u_{\alpha} \otimes v_{\beta}\right)$ in $\mathfrak{A} \widehat{\otimes} \mathfrak{A}$. One of the following cases can occur

(1) $\left(v_{\beta}\right) \subseteq \operatorname{ker} \varphi\left(\right.$ or $\left.\left(u_{\alpha}\right) \subseteq \operatorname{ker} \psi\right)$;

(2) There exists at least one $\beta$ such that $v_{\beta} \notin \operatorname{ker} \varphi$ (or at least for one $\alpha$, $\left.u_{\alpha} \notin \operatorname{ker} \psi\right)$;

(3) There is not any $\beta$ such that $v_{\beta} \in \operatorname{ker} \varphi$ (or no any $\alpha$ that $u_{\alpha} \in \operatorname{ker} \psi$ ). Let $\left(u_{\alpha}\right)$ be bounded by $M_{1} \in(0, \infty)$ and $\left(v_{\beta}\right)$ be bounded by $M_{2} \in(0, \infty)$. If (1) holds, then $\left\langle u_{\alpha} \otimes v_{\beta}, \varphi \otimes \varphi\right\rangle=0$. This implies that

$$
\left\langle u_{\alpha} \otimes v_{\beta}, \varphi \otimes \varphi-\psi\right\rangle=-\varphi\left(u_{\alpha}\right) \psi\left(v_{\beta}\right)=-1
$$

for all $\alpha, \beta$. Thus,

$$
\begin{aligned}
1 & =\left|\left\langle u_{\alpha} \otimes v_{\beta}, \varphi \otimes \varphi-\psi\right\rangle\right| \\
& \leq\left\|u_{\alpha} \mid\right\| v_{\beta}\|\| \varphi\|\| \varphi-\psi \| \\
& \leq M_{1} M_{2}\|\varphi\|\|\varphi-\psi\|
\end{aligned}
$$

for all $\alpha$ and $\beta$. Set $M=\left(M_{1} M_{2}\right)^{-1}$, then $\|\varphi-\psi\| \geq M\|\varphi\|^{-1}$. We consider case $(3)$, because it implies case $(2)$, too. Since $\left(v_{\beta}\right)$ is bounded and $\left|\varphi\left(v_{\beta}\right)\right| \leq$ $\|\varphi\|\left\|v_{\beta}\right\| \leq\|\varphi\| M_{2}$, then we can find a positive number such as $C<\infty$ such that $\left|\varphi\left(v_{\beta}\right)\right|=C \leq\|\varphi\| M_{2}$ for all $\beta$. It follows that

$$
\begin{aligned}
C-1 & =\left|\varphi\left(u_{\alpha}\right) \varphi\left(v_{\beta}\right)\right|-\left|\varphi\left(u_{\alpha}\right) \psi\left(v_{\beta}\right)\right| \\
& \leq\left|\varphi\left(u_{\alpha}\right) \varphi\left(v_{\beta}\right)-\varphi\left(u_{\alpha}\right) \psi\left(v_{\beta}\right)\right| \\
& =\left|\left\langle u_{\alpha} \otimes v_{\beta}, \varphi \otimes \varphi-\psi\right\rangle\right| \\
& \leq\left\|u_{\alpha} \mid\right\| v_{\beta}\|\| \varphi\|\| \varphi-\psi \| \\
& \leq M_{1} M_{2}\|\varphi\|\|\varphi-\psi\|
\end{aligned}
$$

for all $\alpha$ and $\beta$. Set $M=\left(M_{1} M_{2}\right)^{-1}(C-1)$, then $\|\varphi-\psi\| \geq M\|\varphi\|^{-1}$. This completes the proof. 
Let $(X, d)$ be a metric space. A subset $Y$ of $X$ is said to be uniformly discrete if for every distinct $x, y \in Y$, there exists $\varepsilon>0$ such that $d(x, y)>\varepsilon$. By the above theorem, we obtain the following result.

Corollary 2.3. Let $\mathfrak{A}$ be a Banach algebra with carrier space $\sigma(\mathfrak{A})$. If $\mathfrak{A}$ is character amenable, then $\sigma(\mathfrak{A})$ is a uniformly discrete subset of $\mathfrak{A}^{*}$.

Let $X$ be a metric space with metric $d$, and take $\alpha$ such that $0<\alpha \leq 1$. The Lipschitz algebra $\operatorname{Lip}_{\alpha} X$ is the space of complex-valued functions $f$ on $X$ such that

$$
p_{\alpha}(f)=\sup \left\{\frac{|f(x)-f(y)|}{d(x, y)^{\alpha}}: x, y \in X, x \neq y\right\}<\infty .
$$

For each $f \in \operatorname{Lip}_{\alpha} X$, set

$$
\|f\|_{\alpha}=|f|_{X}+p_{\alpha}(f) .
$$

Then, $\left(\operatorname{Lip}_{\alpha} X,\|f\|_{\alpha}\right)$ is a Banach algebra (on $X$ ). The Lipschitz algebra $\operatorname{lip}_{\alpha} X$ is a closed subalgebra of $\operatorname{Lip}_{\alpha} X$ (for more details see [20] and [21]). A function $f \in \operatorname{lip}_{\alpha} X$ if

$$
\frac{|f(x)-f(y)|}{d(x, y)^{\alpha}} \rightarrow 0 \quad \text { as } d(x, y) \rightarrow 0 .
$$

Many results on amenability and weak amenability of Lipschitz algebras are given in $[3,7,8]$. For any $\varphi \in \sigma\left(\operatorname{Lip}_{\alpha} X\right)\left(\varphi \in \sigma\left(\operatorname{lip}_{\alpha} X\right)\right), \varphi$-amenability of $\operatorname{Lip}_{\alpha} X\left(\operatorname{lip}_{\alpha} X\right)$, considered by Kaniuth and et al. (see [14, Example 5.3]), and some results about character amenability of these algebras are studied in $[4,11]$.

Let $(X, d)$ be uniformly discrete. Then, Lipschitz norm is equivalent to the uniform norm. Thus, $\operatorname{Lip}_{\alpha} X=\operatorname{lip}_{\alpha} X=C(X)=C(\beta X)$, where $\beta X$ is Ston-Cech compactification of $X$. To prove the following theorem, we use the technique of Theorem 3 of [7], and we characterize the character amenability of Lipschitz algebras as follows.

Theorem 2.4. Let $(X, d)$ be a metric space. Then, the following statements are equivalent:

(i) $\operatorname{Lip}_{\alpha} X\left(\right.$ lip $\left._{\alpha} X\right)$ is character amenable;

(ii) $(X, d)$ is uniformly discrete;

(iii) $\operatorname{Lip}_{\alpha} X\left(\operatorname{lip}_{\alpha} X\right)$ is amenable.

Proof. We prove the result for $\operatorname{Lip}_{\alpha} X$, and for $\operatorname{lip}_{\alpha} X$, the method is similar. Let $\operatorname{Lip}_{\alpha} X$ be character amenable. For $x \in X$, consider the operator $\varphi_{x}$ : $\operatorname{Lip}_{\alpha} X \longrightarrow \mathbb{C}$ with $\varphi_{x}(f)=f(x)$, for all $f \in \operatorname{Lip}_{\alpha} X$. For each character $\varphi$ on $\operatorname{Lip}_{\alpha} X$, there exists a $x \in X$ such that $\varphi=\varphi_{x}$. Then, by operator norm on $\sigma\left(\operatorname{Lip}_{\alpha} X\right)$, we have

$$
\left\|\varphi_{x}-\varphi_{y}\right\|=\sup _{\|f\| \leq 1}\left|\varphi_{x}(f)-\varphi_{y}(f)\right|=\sup _{\|f\| \leq 1}|f(x)-f(y)| \leq d(x, y)^{\alpha}
$$


for all $x, y \in X$. Now, we continue by contradiction. If $(X, d)$ is not uniformly discrete, then by $(2.12), \sigma\left(\operatorname{Lip}_{\alpha} X\right)$ is not uniformly discrete. But this is a contradiction with Corollary 2.3. Therefore, $(X, d)$ is uniformly discrete.

Conversely, suppose that $(X, d)$ is uniformly discrete. Therefore it is sufficient that we consider character amenability of Banach algebra $C(K)$, where $K$ is a compact Hausdorff space. The amenability of $C(K)$ is known (see $[10$, Theorem 5.1.87] or [1, The Main Theorem]). This implies that $\operatorname{Lip}_{\alpha} X$ and $\operatorname{lip}_{\alpha} X$ are character amenable.

For amenability, we refer to Theorem 3 of [7].

Acknowledgment. The authors would like to express their sincere thanks to the referee to the referee for her/his invaluable comments for improving of this paper.

\section{References}

[1] M. Abtahi and Y. Zhang, A new proof of the amenability of $C(X)$, Bull. Aust. Math. Soc. 81 (2010), no. 3, 414-417.

[2] M. Alaghmandian, R. Nasr-Isfahani, and M. Nemati, Character amenability and contractibility of abstract Segal algebras, Bull. Aust. Math. Soc. 82 (2010), no. 2, 274-281.

[3] W. G. Bade, P. C. Curtis, and H. G. Dales, Amenability and weak amenability for Beurling and Lipschitz algebras, Proc. London Math. Soc. 55 (1987), no. 2, 359-377.

[4] M. Dashti, R. Nasr-Isfahani, and S. Soltani Renani, Character Amenability of Lipschitz Algebras, Canad. Math. Bull. 57 (2014), no. 1, 37-41.

[5] A. Ghaffari, On character amenability of semigroup algebras, Acta Math. Hungar. 134 (2012), no. 1-2, 177-192.

[6] F. Ghahramani and R. J. Loy, Generalized notion of amenability, J. Funct. Anal. 208 (2004), no. 1, 229-260.

[7] F. Gourdeau, Amenability of Banach algebras, Math. Proc. Cambridge Phil. Soc. 105 (1989), no. 2, 351-356.

[8] , Amenability of Lipschitz algebras, Math. Proc. Cambridge Phil. Soc. 112 (1992), no. 3, 581-588.

[9] N. Grønbæk, Amenability and weak amenability of tensor algebras and algebras of nuclear operators, J. Aust. Math. Soc. 51 (1991), no. 3, 483-488.

[10] A. Ya. Helemskii, Banach and Locally Convex Algebras, Oxford University Press, Oxford, 1993.

[11] Z. Hu, M. Sangani Monfared, and T. Traynor, On character amenable Banach algebras, Studia Math. 193 (2008), no. 1, 53-78.

[12] B. E. Johnson, Cohomology in Banach algebras, Memoirs of the American Mathematical Society, No. 127. American Mathematical Society, Providence, R.I., 1972.

[13] E. Kaniuth, A. T. Lau, and J. Pym, On $\varphi$-amenability of Banach algebras, Math. Proc. Camb. Phil. Soc. 144 (2008), no. 1, 85-96.

[14] _ On character amenability of Banach algebras, J. Math. Anal. Appl. 344 (2008), no. 2, 942-955.

[15] A. T.-M. Lau, Characterization of amenable Banach algebras, Proc. Amer. Math. Soc. 70 (1978), 156-160.

[16] - Analysis on a class of Banach algebras with application to harmonic analysison locally compact groups and semigroups, Fund. Math. 118 (1983), 161-175.

[17] A. T.-M. Lau and Y. Zhang, Finite dimensional invariant subspace property and amenability for a class of Banach algebras, Trans. Amer. Math. Soc., to appear 
[18] M. S. Monfared, Character amenability of Banach algebras, Math. Proc. Cambrigde Phil. Soc. 144 (2008), no. 3, 697-706.

[19] R. Nasr-Isfahani and M. Nemati, Essential character amenability of Banach algebras, Bull. Aust. Math. Soc. 84 (2011), no. 3, 372-386.

[20] D. R. Sherbert, The structure of ideals and point derivations in Banach algebras of Lipschitz functions, Trans. Amer. Math. Soc. 111 (1964), 240-272.

[21] N. Weaver, Lipschitz Algebras, World Scientific Publishing Co. Pte Ltd., 1999.

Madjid Eshaghi Gordji

Department of Mathematics

SEmnan University

P. O. Box 35195-363, SEmnan, Iran

E-mail address: madjid.eshaghi@gmail.com, meshaghi@semnan.ac.ir

Ali JABBARI

Young Researchers and Elite Club, Ardabil Branch

ISLAMIC AZAD UNIVERSITY

ARDABIL, IRAN

E-mail address: jabbari_al@yahoo.com, ali.jabbari@iauardabil.ac.ir

GWANG Hui Kim

Department of Mathematics

KANGNAM UNIVERSITY

YONGIN 446-702, KorEA

E-mail address: ghkim@kangnam.ac.kr 